\title{
Implantación de la Docencia Inversa en las prácticas de la asignatura "Teoría y Diseño de Máquinas"
}

\author{
Sergi Montava Jordà a*, Samuel Sánchez Caballeroa, Miguel Ángel Sellés Cantóa, Antonio Vicente \\ Martinez Sanz ${ }^{\text {a }}$ Vicente Colomer Romero ${ }^{a}$ \\ ${ }^{a}$ Departamento de Ingeniería Mecánica y de Materiales, Escuela Politécnica Superior de Alcoy. Universitat \\ Politècnica de València (sermonjo@mcm.upv.es ${ }^{*}$, sasanca@dimm.upv.es,$\underline{\text { maselles@dimm.upv.es }}$, \\ anmarsan@mcm.upv.es, vicoro@mcm.upv.es)
}

\begin{abstract}
In this work both the initiative and the results of the implementation of flipped classroom in the practices of the course Theory and Design of Machines given in the 3rd year of the Mechanical Engineering Degree are presented. Since the 2014-2015 academic year, the UPV has been promoting the implementation of this methodology with the aim of improving learning by students and this has been the main purpose of this work. This type of methodology implies a greater involvement and commitment of both teachers and students, and the latter must adopt a more active and participatory role. The result of the implementation of this initiative has been positive on the part of the teaching staff given that despite having had to dedicate extra dedication to the elaboration of the material that the methodology demanded, the academic results have improved, although on the part of the students the feeling It has not been as good as most have seen an excessive workload in addition to the difficulties involved in changing methodologies.
\end{abstract}

Keywords: Reverse teaching, flipped classroom, teaching innovation, machine design, active learning, autonomous learning

\section{Resumen}

En el presente trabajo se presentan tanto la iniciativa como los resultados de la puesta en marcha de la docencia inversa en las prácticas de la asignatura Teoría y Diseño de Máquinas impartida en el $3^{\circ}$ curso del Grado de Ingeniería Mecánica. Desde el curso académico 2014-2015 la UPV se está impulsando la implantación de esta metodología con el objetivo de mejorar el aprendizaje por parte de los alumnos y este ha sido la principal finalidad de este trabajo. Este tipo de metodología supone una mayor implicación y compromiso tanto de los profesores como de los alumnos debiendo adoptar estos últimos un rol más activo y participativo. El resultado de la implantación de esta iniciativa ha sido positiva por parte del profesorado dado que a pesar de haber tenido que haber dedicar una dedicación extra para la elaboración del material que la metodología exigía los resultados académicos han mejorado, aunque por parte del alumnado la sensación no ha sido tan buena puesto que la mayoría ha visto una carga de trabajo excesiva además de las dificultades que implican los cambios de metodologías.

Palabras clave: Docencia inversa, aula inversa, innovación docente, diseño de máquinas, aprendizaje activo, aprendizaje autónomo 


\section{Introducción}

Con la incorporación de las universidades españolas dentro del Espacio Europeo de Educación Superior (EEES) se ha buscado mejorar de la competitividad internacional entre las universidades de la Unión Europea y para conseguirlo se requiere centrar la atención sobre el alumno (Garcia et al., 2017). Para conseguir este objetivo se necesitan incorporar metodologías más activas que fomenten la participación del alumno en el proceso de enseñanza-aprendizaje que fomenten el protagonismo de este (Sánchez, 2011). En la actualidad existen diferentes metodologías en auge, más activas que las tradicionales con las que además se obtienen mejores resultados académicos de los alumnos, como son: el Flipped Classroom, el Aprendizaje Basado en Proyectos (ABP), el Aprendizaje Cooperativo, la Gamificación, el Aprendizaje basado en problemas, el Design Thinking (DT), el Aprendizaje Basado en el Pensamiento (Thinking Based Learning) o el Aprendizaje Basado en Competencias.

En el presente trabajo nos hemos centrado en la metodología Flipped Classroom, aula invertida o Docencia Inversa que es un modelo pedagógico que transfiere el trabajo de determinados procesos de aprendizaje más sencillos, simples o concretos fuera del aula y utiliza el tiempo de clase, junto con la experiencia del docente, para facilitar, interactuar y potenciar otros procesos de adquisición y práctica de conocimientos de mayor nivel cognitivo dentro del aula o simplemente actuando como guía de los alumnos para poder resolver dudas (FLIPPED CLASSROOM, 2019).

Se trata de un enfoque integral que combina la instrucción directa con métodos constructivistas, el incremento de compromiso e implicación de los estudiantes con el contenido del curso y mejorar su comprensión conceptual. Este enfoque integral que, cuando se aplica con éxito, apoyará todas las fases de un ciclo de aprendizaje (Taxonomía de Bloom) donde los dos niveles inferiores se trabajaran en casa, mientras que a partir del tercer nivel se trabajará en clase (UPV Docencia Inversa, 2014) como se puede apreciar en la Figura 1.

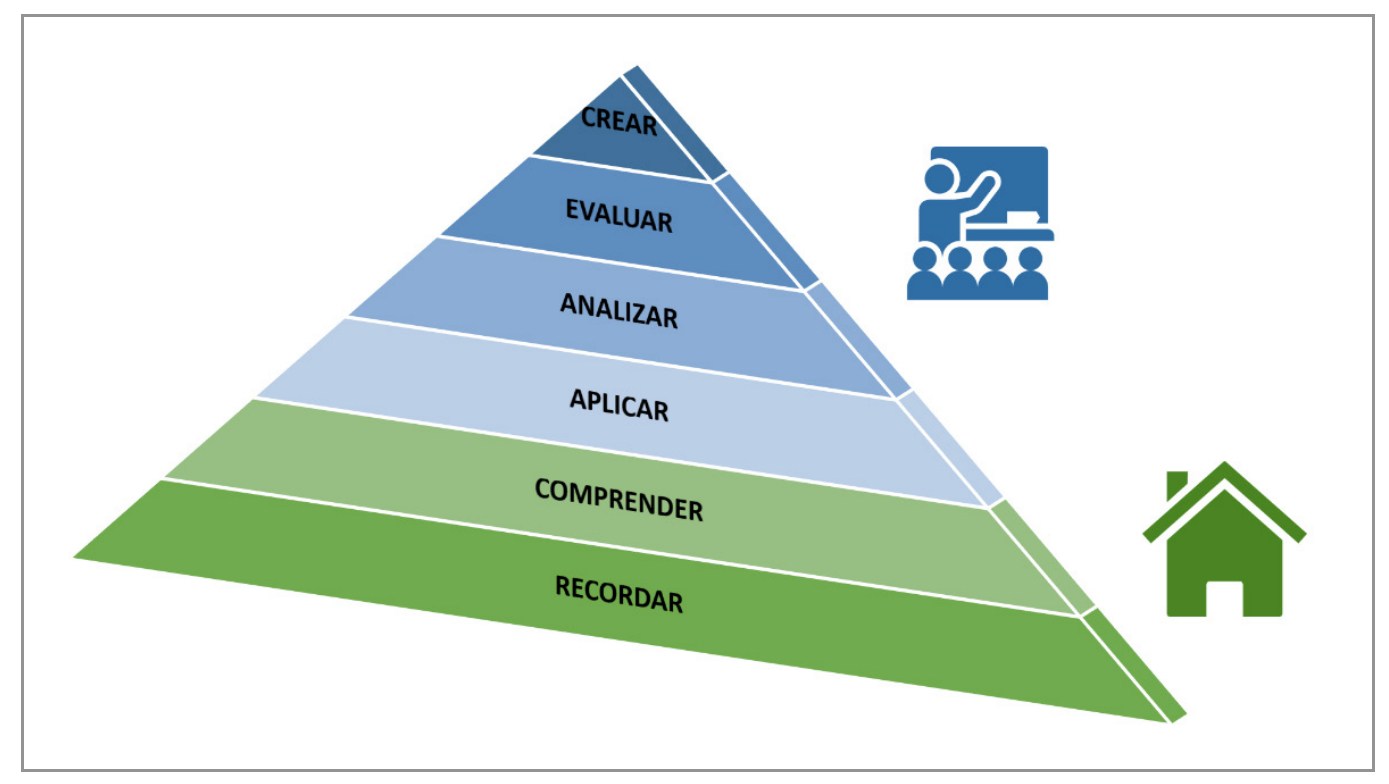

Fig. 1 Niveles de la taxonomía de Bloom 
Los orígenes de esta metodología surgieron en 2007 por Jonathan Bergmann y Aaron Sams, dos profesores de química del instituto Woodland Park en Colorado (EE.UU.), que lo bautizaron con el nombre "Flipped Classroom". Bergmann y Sams se dieron cuenta de que los estudiantes frecuentemente perdían algunas clases y para ayudar a estos alumnos, impulsaron la grabación y distribución de video, pero además, se dieron cuenta que este mismo modelo permite que el profesor centre más la atención en las necesidades individuales de aprendizaje de cada estudiante (Sams y Bergmann, 2013).

Entre las principales ventajas de la docencia inversa frente a la docencia tradicional cabe destacar (FLIPPED CLASSROOM, 2019):

- Permite a los docentes dedicar más tiempo a la atención a la diversidad.

- Permite al profesorado compartir información y conocimiento entre sí, con el alumnado

- Proporciona al alumnado la posibilidad de volver a acceder a los mejores contenidos generados o facilitados por sus profesores, adaptándose al ritmo de cada estudiante

- Crea un ambiente de aprendizaje colaborativo en el aula, disminuyendo el absentismo.

- Involucra al alumnado desde el inicio del proceso de aprendizaje, transfiriendo más y mejor.

\section{Objetivos}

El objetivo principal de este trabajo es mostrar los resultados académicos de los alumnos así como las opiniones de estos tras implantar la metodología de la Docencia Inversa en las sesiones prácticas de la asignatura Teoría y Diseño de Máquinas, para poder realizar una toma de decisiones en la estrategia a seguir y valorar los beneficios de esta metodología.

A partir de este objetivo principal surgen otros objetivos específicos como:

- Analizar y evaluar los resultados obtenidos por parte de los alumnos en los apartados prácticos.

- Analizar y evaluar la opinión de los alumnos con la ayuda de encuesta de satisfacción anónima realizada con la aplicación Formularios de Google.

En los siguientes apartados se presentan tanto las características principales de la asignatura, la metodología docente, el sistema de evaluación empleado y el material docente realizado expresamente para la innovación. En el apartado de resultados se mostrarán tanto la evolución de los resultados académicos hasta la aplicación de la innovación como los de la encuesta de satisfacción. Por último, se incluyen las conclusiones de la iniciativa así como futuras líneas de trabajo.

\section{Desarrollo de la innovación}

\subsection{Descripción de la asignatura}

La asignatura Teoría y Diseño de Máquinas (en adelante TDM) en la que se centra este trabajo es de carácter obligatorio y anual del tercer curso del Grado de Ingeniería Mecánica de la Universitat Politècnica de València. Según el plan de estudios actual (BOE 16-03-2011) consta de un total de 9,0 créditos ETCS (European Credits Transfer System) repartidos en 4,5 créditos para la parte teórica y 4,5 créditos para la práctica, por tanto, para poder superar la asignatura son igual de importantes ambas partes (Montava et al.,2019). 
Durante el curso que se ha decidido implantar la iniciativa 2018-2029 se han matriculado 129 alumnos repartidos en cuatro grupos de prácticas con más de 30 alumnos por grupos con un total de 22 sesiones de prácticas de laboratorio de 2 horas por grupo.

\subsection{Metodología docente}

Aprovechando que la UPV incentiva la participación en el proyecto "DOCENCIA INVERSA" dentro del PLAN DE INNOVACIÓN EDUCATIVA A+D, se optó por participar y seguir tanto el programa como los objetivos marcados por el Vicerrectorado de Recursos Digitales y Documentación.

Se marcaron unos ítems de obligado cumplimiento como (UPV Vicerrectorado de Recursos Digitales y Documentación, 2018):

- Utilización de la herramienta LESSONS de PoliformaT.

- Sesión de presentación de la metodología a los alumnos.

- Participación de los alumnos en la encuesta.

- Participación del profesor en la encuesta.

- Implantación efectiva de la metodología (programación de las actividades en el aula y en casa).

Respecto al grado de implicación de la metodología en la no se exigen ningún porcentaje en concreto pero teniendo en cuenta que era el primer año se optó por implantarla en las 5 primeras sesiones solamente lo cual supone cerca de un $23 \%$ de las sesiones totales.

Según la guía de la implantación de la Docencia Inversa UPV (UPV ASIC - ICE, 2018) implantar esta metodología supone un sobre gran esfuerzo por parte del profesorado puesto que hay que realizer una serie de tareas previas como:

- En la presentación de la metodología se debe preparar al alumno a este nuevo modo de trabajo informándoles sobre: En qué consiste la metodología; Qué se espera de ellos y Qué valor le va a aportar a su aprendizaje.

- En el plan de trabajo se debe especificar al alumno de manera esquemática de: Resultados de aprendizaje; Actividades a realizar: Previas a la sesión (en casa) Durante la sesión (en el aula) y Tras la sesión (en casa); Criterios de seguimiento y evaluación.

- En la descripción de las actividades de aprendizaje a realizar por el alumno: antes, durante y después de la sesión de aula inversa, se realizará una ficha para cada una de las actividades con las explicaciones suficientes facilitando el trabajo autónomo por parte del alumno.

\subsection{Sistema de evaluación}

Aprovechando que en el curso anterior se implanto el sistema de tareas semanales mediante la plataforma PoliformaT se decidió evaluar las tareas realizadas con la Docencia Inversa también dentro del ítem PARTICIPACIÓN EN CLASE, de forma que las sesiones que disponían de SESIÓN INVERSA no presentarían actividades semanales. La contribución activa por parte del alumno supondría por tanto hasta un $10 \%$ de la nota final de la asignatura.

\subsection{Elaboración de material docente}

Cabe destacar que los materiales empleados en la docencia inversa son una de las claves principales para el buen funcionamiento y para la obtención de buenos resultados. Por este motivo es muy importante la

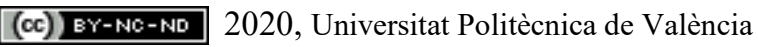

Congreso In-Red (2020) 
calidad de los materiales en línea de los que dispone el alumno para trabajar de forma autónoma y estar dispuestos a evaluarlos buscando evidencias que muestren la calidad de estos para mejorar la calidad de estos se deben estudiar evidencias que muestren la calidad de dichos materiales empleados para la mejora continua de la iniciativa (Bas et al.,2017).

\subsubsection{Secuencias didácticas desarrolladas en Lessons de poliformaT}

A partir de las recomendaciones de la "Guía de implantación de la DOCENCIA INVERSA UPV" se plasmó con la herramienta LESSONS de PoliformaT tanto la presentación como la programación detallada, la cual permitirá guiar a los alumnos durante el curso. En la Figura 2 se aprecia el aspecto de la página de LESSONS preparada para la presentación tanto de la metodología como del plan de trabajo.

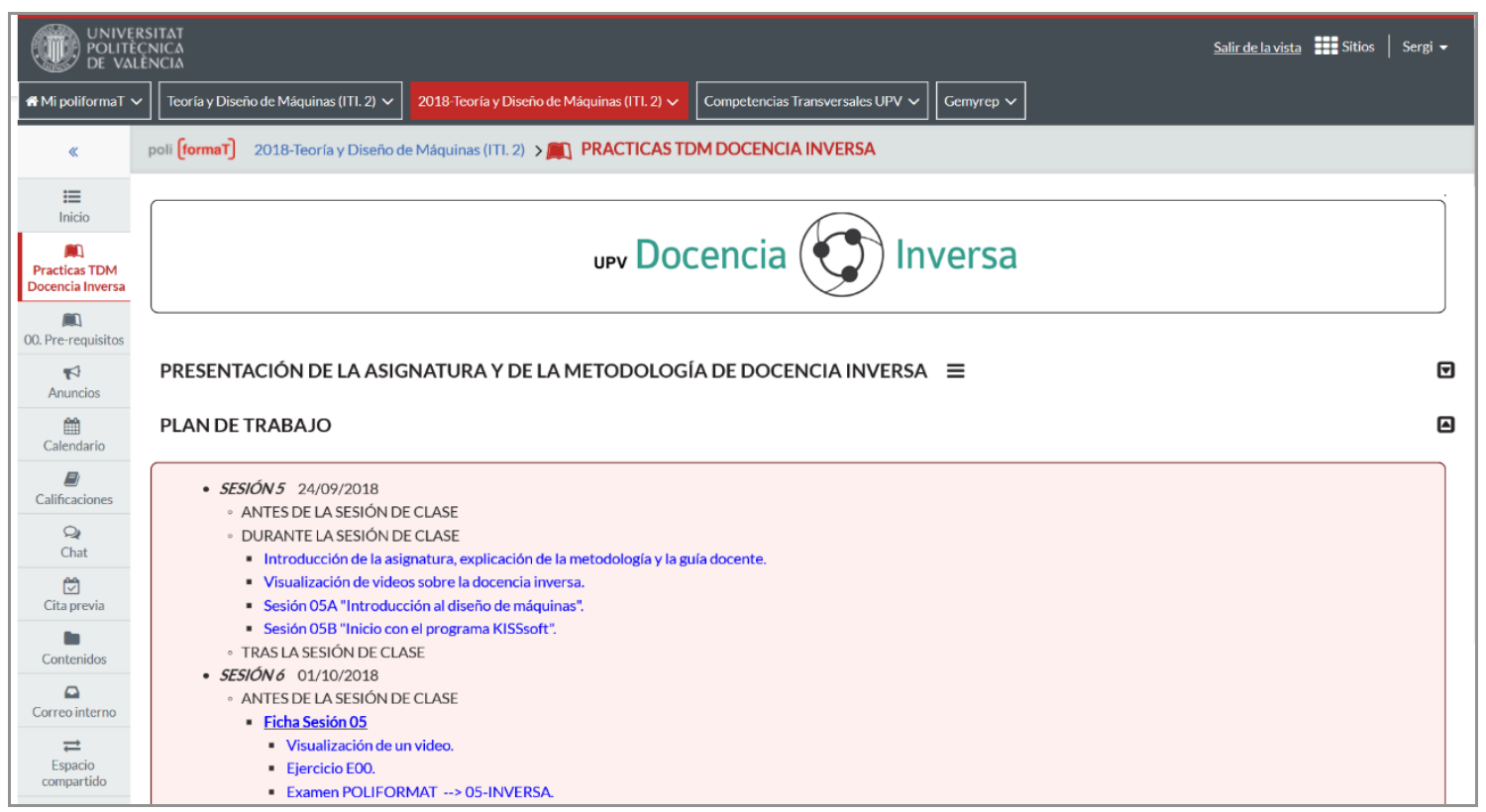

Fig. 2 Vista de la presentación de la metodología de la Docencia Inversa en LESSONS.

\subsubsection{Dinámicas de aula desarrolladas y fundamentadas}

Se realizaron para cada una de las sesiones inversas una ficha de las actividades de aprendizaje diferenciando el trabajo previo en casa como el trabajo a realizar en el aula. Además nos permite se indicar: la duración estimada, el resultado de aprendizaje, los contenidos que se trabajan y las actividades como diferentes explicaciones, así como si fuese necesario algún tipo de link para abrir una página web o video de internet.

En concreto se prepararon las 5 primeras sesiones iniciales de la asignatura diferentes actividades como:

- Modelado de un árbol de transmisión en un software CAD

- Realización de un plano de fabricación de un árbol de transmisión

- Realización de una hoja de cálculo para la obtención de los datos de resistencia de una viga.

- Introducción de los datos básicos de un cálculo de árbol con el software CAE.

- Cálculo y análisis de los resultados de un cálculo de árbol con el software CAE. 
En la Figura 3 se puede ver una de las fichas de aprendizaje de las sesiones con docencia inversa donde podemos apreciar tanto el trabajo precio en casa como el trabajo a realizar en el aula.

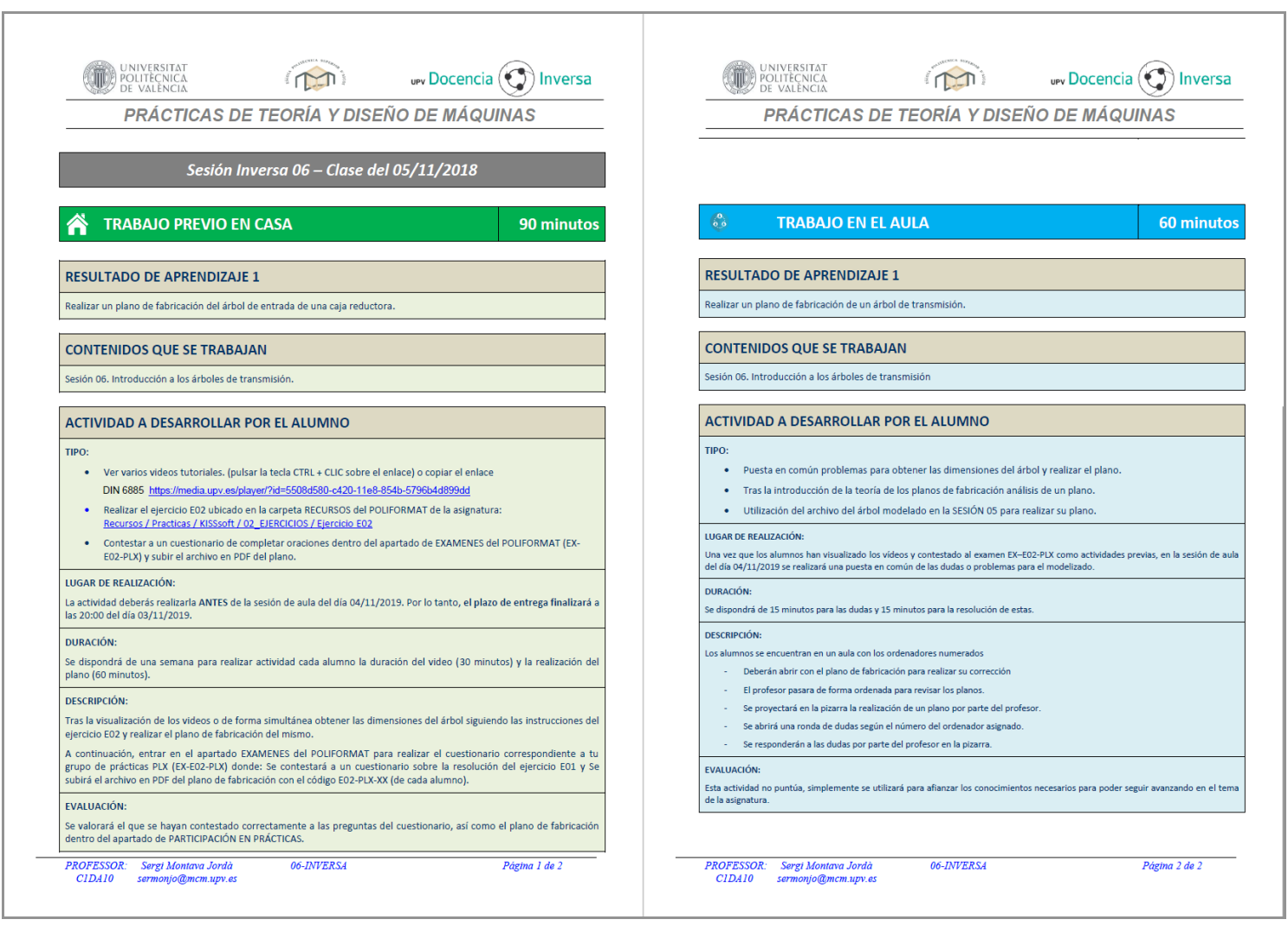

Fig. 3 Ejemplo de una de las fichas de actividades de aprendizaje.

\subsubsection{Videos}

A pesar que la Universitat Politècnica de València dispone de una gran cantidad de videos disponibles desde su repositorio de videos UPV [Media] (https://media.upv.es) o incluso de los disponibles en internet en plataformas como YouTube (https://www.youtube.com), se decidió el preparar una serie de videos más enfocados en las propias actividades planteadas a pesar que después se subieron al repositorio de la universidad. En las Figura 4 podemos apreciar algunos de los videos que se prepararon dentro del repositorio UPV [Media] y en la Figura 5 uno de los videos preparados para la Sesión Inversa - 06. 


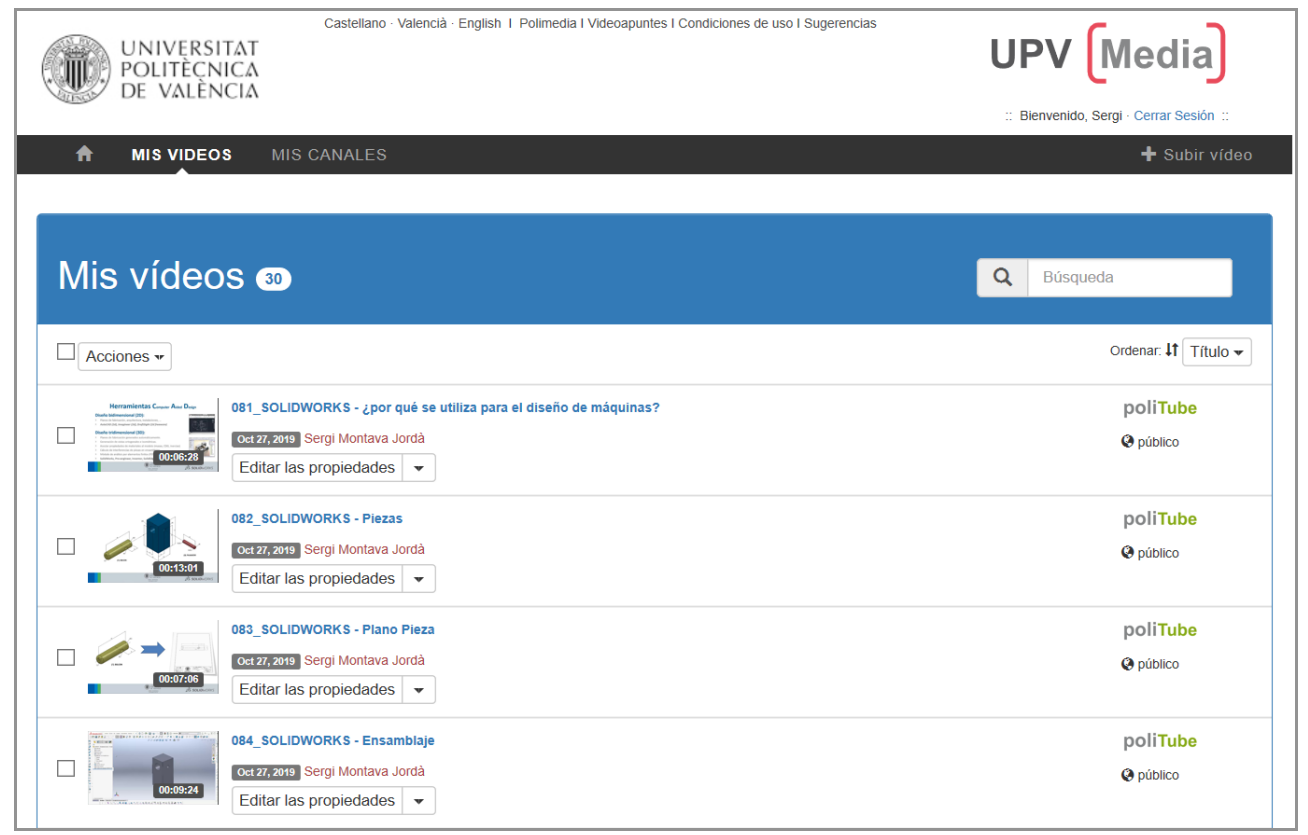

Fig. 4 Ejemplo de los videos preparados dentro del repositorio UPV [Media].

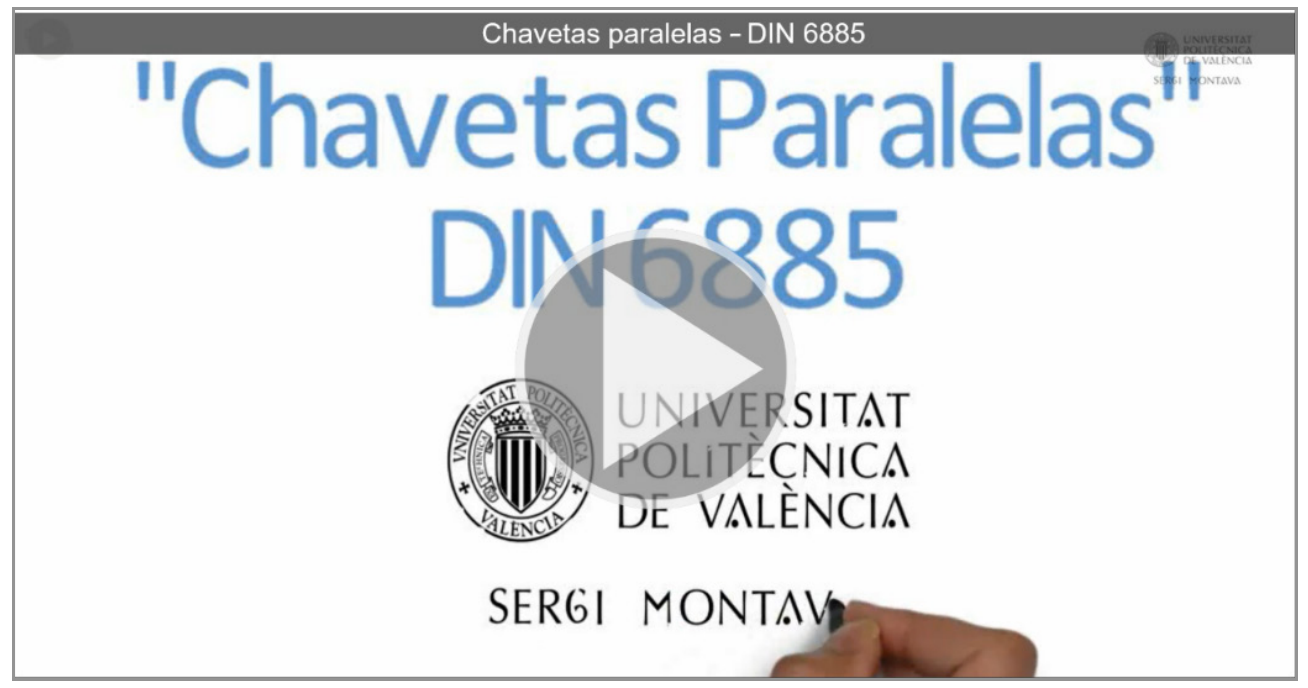

Fig. 5 Ejemplo de uno de los videos preparados para la Sesión Inversa - 06.

\section{Resultados}

\subsection{Resultados académicos}

Durante los últimos cursos en la asignatura TDM el nivel de aprobados ha ido descendiendo poco a poco como se puede apreciar tanto en la Tabla1 como en la Figura 6. Tras una reflexión por parte del profesorado se intentó buscar soluciones a este problema por un lado planteo la implantación de tareas semanales y por otro lado la implantación de la "Docencia Inversa". Aparentemente, parece que en el curso 2018-2019 de implantación de las medidas se han notado cambios en el porcentaje de aprobados pasando de un $61 \%$ en el curso anterior a un nivel del $71 \%$ en el curso de estudio. 
Tabla 1. Resultados académicos de los últimos cursos de la asignatura TDM.

\begin{tabular}{ccccc}
\hline CURSO & MATRICULADOS & APROBADOS & SUSPENDIDOS & NO PRESENTADOS \\
\hline $2014-2015$ & 124 & 94 & 29 & 1 \\
$2015-2016$ & 123 & 90 & 33 & 0 \\
$2016-2017$ & 97 & 78 & 19 & 0 \\
$2017-2018$ & 115 & 70 & 45 & 0 \\
$2018-2019$ & 129 & 91 & 38 & 0 \\
\hline
\end{tabular}

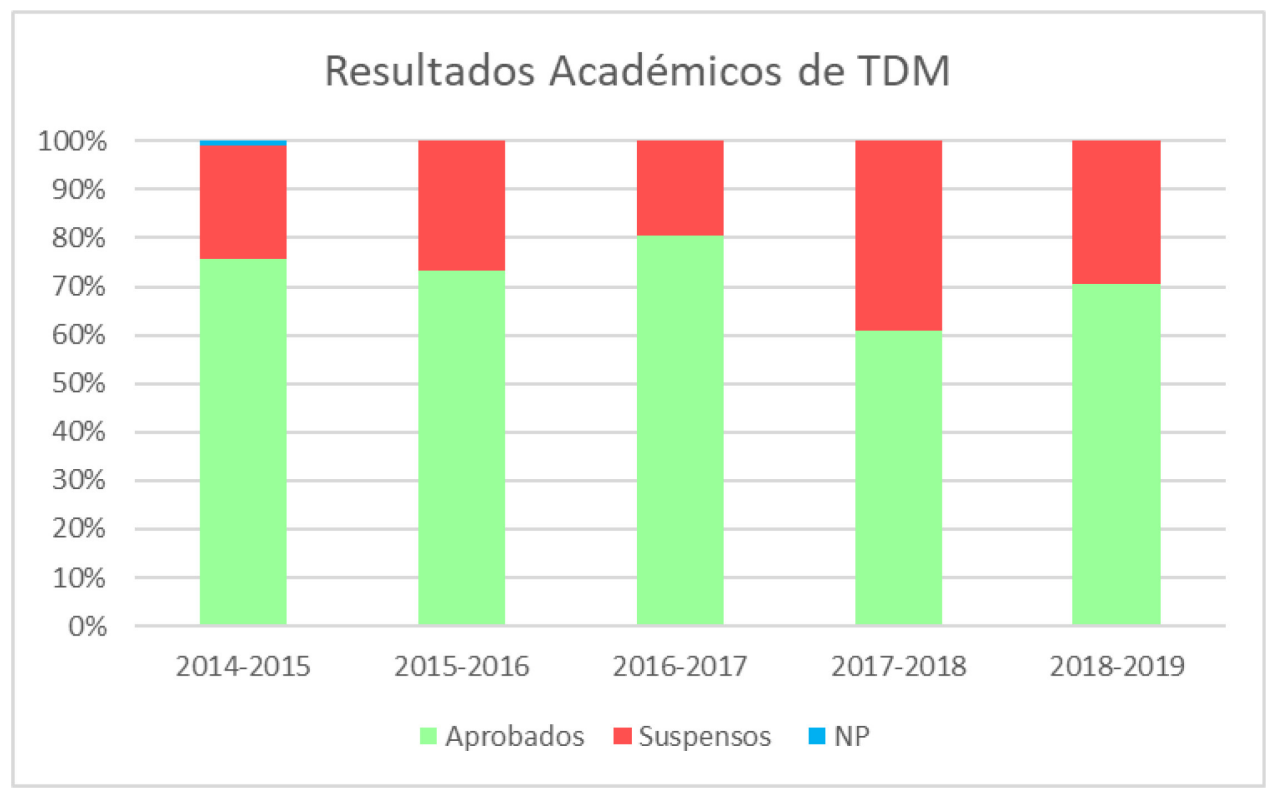

Fig. 6 Resultados académicos de los últimos cursos de la asignatura TDM.

\subsection{Resultados de la encuesta de satisfacción}

Además de la encuesta realizada por el Vicerrectorado de Recursos Digitales y Documentación al participar dentro del proyecto "Docencia Inversa" también se les hizo realizar a los alumnos una encuesta de satisfacción con la ayuda de la aplicación Formularios de Google. Esta encuesta se realizó en la siguiente clase tras la finalización de las sesiones en las que se aplicó la metodología de docencia inversa la cual consta de breves preguntas que se detallaran a continuación para tener un feedback más realista de cuál era la percepción de los alumnos sobre esta experiencia. Respecto a la tasa de participación en la encuesta fue muy representativa, alrededor del $88,9 \%$ del total de alumnos matriculados, ya que las encuestas no contestadas eran asistentes no habituales de las sesiones prácticas. 
En la Figura 7 se observan los resultados a la pregunta: ¿Qué tipo de metodología docente preferirías que se utilizará en las prácticas de la asignatura? Donde se aprecia que casi un $64 \%$ de los encuestados casi dos tercios del total preferían la metodología tradicional, lo cual puede ser debido a la resistencia al cambio que presentan los alumnos por el cambio de metodología como se ha apreciado en otros proyectos (Benítez y García, 2013).

¿Qué tipo de metodologia docente preferirias que se utilizará en las prácticas de la asignatura?

113 respuestas

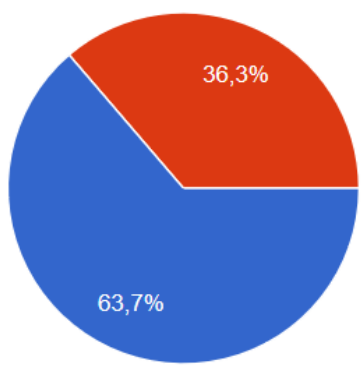

El método tradicional

La metodología de docencia inversa

Fig. 7 Respuestas a la pregunta: ¿Qué tipo de metodología docente preferirías que se utilizará en las prácticas de la asignatura?

En la Figura 8 se observan los resultados a la pregunta: ¿Te gustaría tener más asignaturas en las que se aplicara el método de docencia inversa? Casi la mitad de los encuestados en concreto un $46 \%$ no están a favor de reaplicar la metodología en otras asignaturas, de nuevo este elevado porcentaje se debe a la resistencia al cambio.

¿Te gustaría tener más asignaturas en las que se aplicara el método de docencia inversa?

113 respuestas

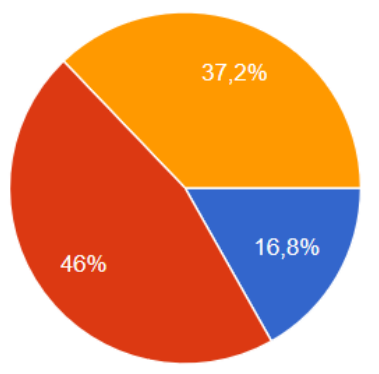

Fig. 8 Respuestas a la pregunta: ¿Te gustaría tener más asignaturas en las que se aplicara el método de docencia inversa?

En la Figura 9 se observan los resultados a la pregunta: ¿Te han parecido útiles y adecuados los materiales docentes y vídeos entregados para trabajar la asignatura en casa? Donde la puntuación 5 
equivale a MUCHO y la de 1 equivale a NADA, se puede ver como a casi el $40 \%$ les ha gustado mucho y a un $12 \%$ les gustó MUCHO lo cual nos da a entender que el material está bastante bien pero se podría mejorar, esta información es útil para los docentes para la realización de futuras actividades a realizar.

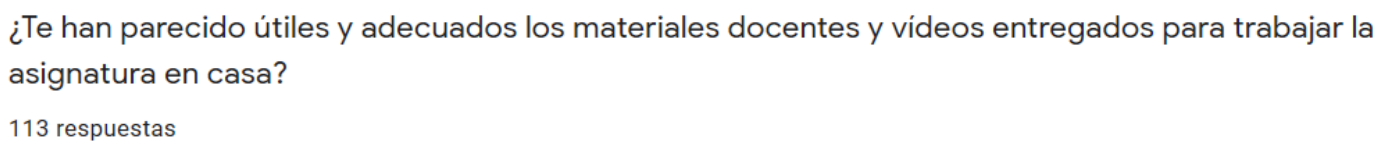

60

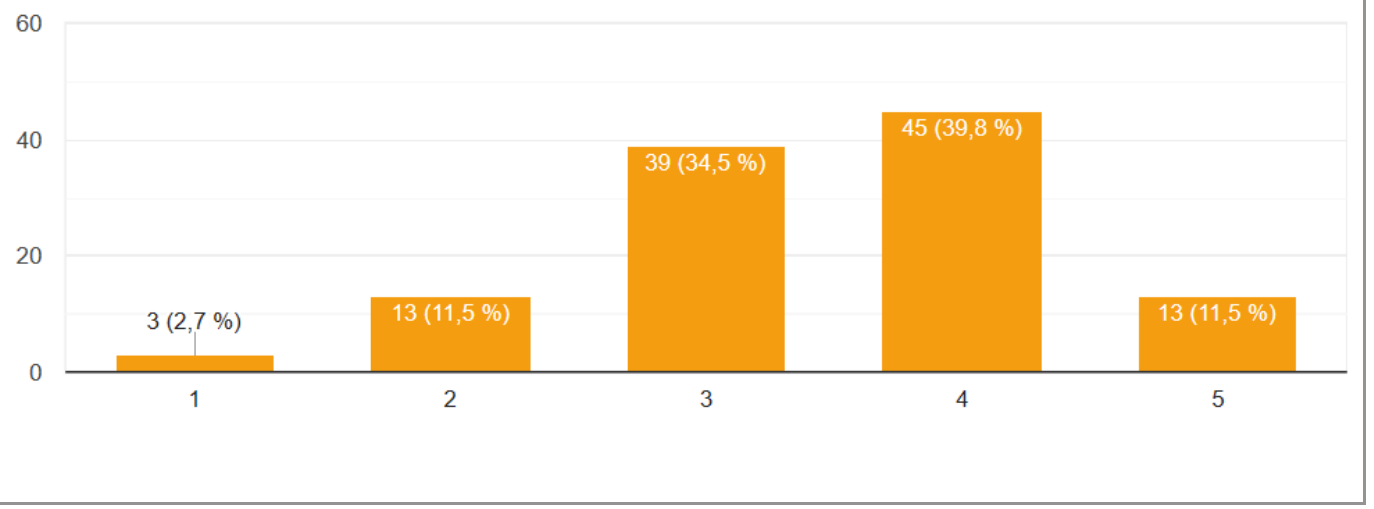

Fig. 9 Respuestas a la pregunta: ¿Te han parecido útiles y adecuados los materiales docentes y videos entregados para trabajar la asignatura en casa?

En la Figura 10 se observan los resultados a la pregunta: ¿Has realizado las actividades planteadas para realizar en casa antes de las clases? Donde se puede apreciar que el 66\% dos tercios de los encuestados han participado o tenían interés en participar en la implantación de la metodología, lo cual confirma que aproximadamente un tercio de los matriculados ni tienen mucho interés por la asignatura.
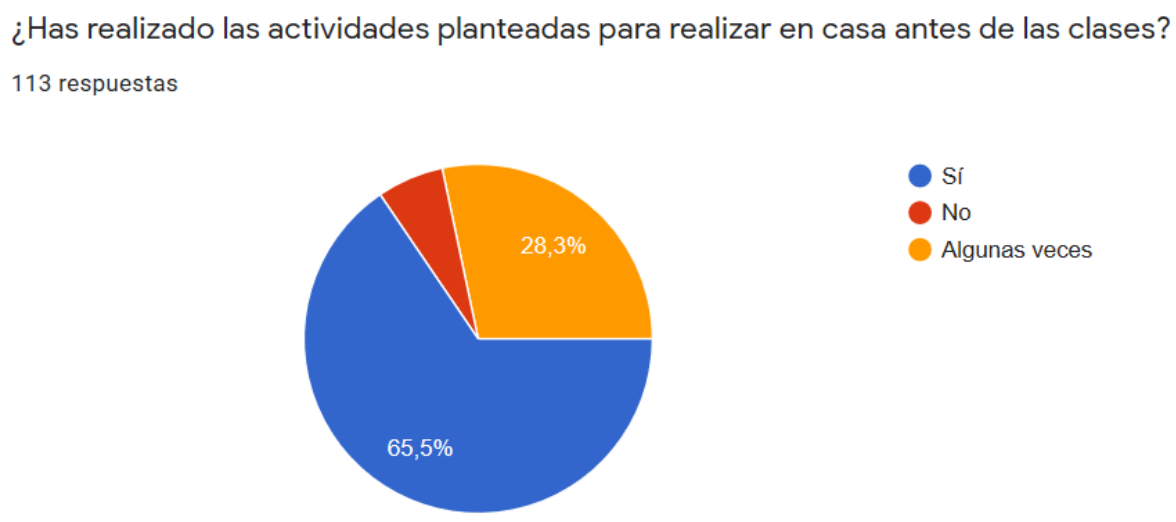

Fig. 10 Respuestas a la pregunta: ¿Has realizado las actividades planteadas para realizar en casa antes de las clases?

En la Figura 11 se observan los resultados a la pregunta: ¿Cuál ha sido el motivo de su realización o no de estas actividades? Como era de esperar casi la mitad un $48 \%$ han participado en la iniciativa porque tenía una recompensa en la nota final, al $15 \%$ les ha faltado tiempo para realizar las actividades, al $2 \%$ no han 
considerado realizarlas y el resto un 35\% aproximadamente han considerado otros motivos y a nadie le ha parecido no interesante realizarlos. Como era de esperar, la mayoría de los alumnos que realizaron las actividades ha sido porque puntuaban para la calificación final de la asignatura y nos lleva a pensar que a mayor recompensa mayor participación.

¿Cuál ha sido el motivo de su realización o no de estas actividades?

113 respuestas

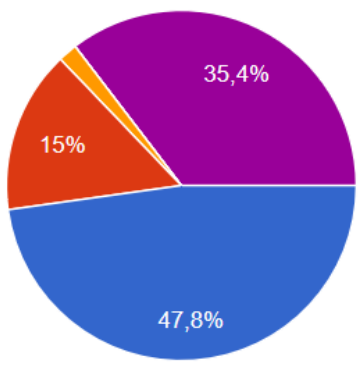

Porque puntua para la nota final de la signatura

Falta de tiempo

No lo he considerado necesario

No me parece interesante

Otros motivos

Fig. 11 Respuestas a la pregunta: ¿Cuál ha sido el motivo de su realización o no de estas actividades?

En la Figura 12 se observan los resultados a la pregunta: ¿Te han parecido interesantes las siguientes actividades? Las opiniones han sido muy buenas puesto que la mayoría de las actividades han parecido interesantes a más del $80 \%$ a excepción de la realización de la hoja de cálculo donde a tan solo un $47 \%$ le ha parecido interesante, esto nos puede dar una información valiosa para identificar qué tipo de actividades gustan más que otras y ayuda a la realización de futuras actividades.

¿Te han parecido interesantes las siguientes actividades?

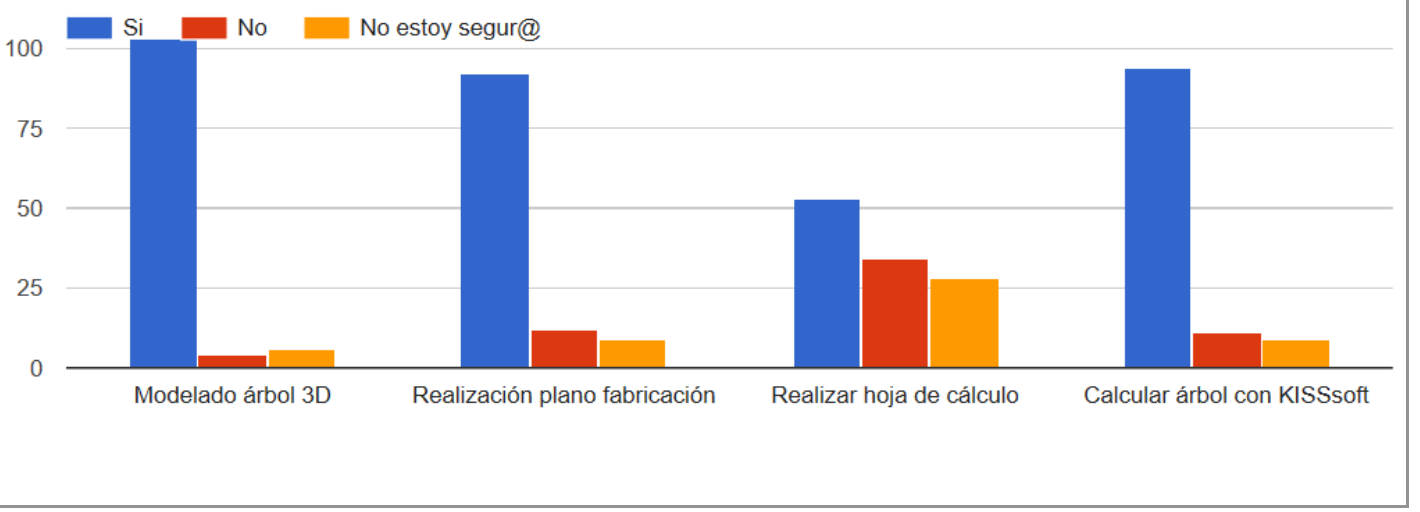

Fig. 12 Respuestas a la pregunta: ¿Te gustaría que repitiera la experiencia en más sesiones? 
En la Figura 13 se observan los resultados a la pregunta: ¿Te han parecido útiles y adecuadas las actividades realizadas en clase, para corregir o afianzar conocimientos? Donde la puntuación 5 equivale a MUCHO y la de 1 equivale a NADA, se puede ver como hay más dispersión de opiniones y tan solo a casi un $20 \%$ no le han parecido NADA o POCO útiles y adecuadas las actividades.

¿Te han parecido útiles y adecuadas las actividades realizadas en clase, para corregir o afianzar conocimientos?

113 respuestas

60

40

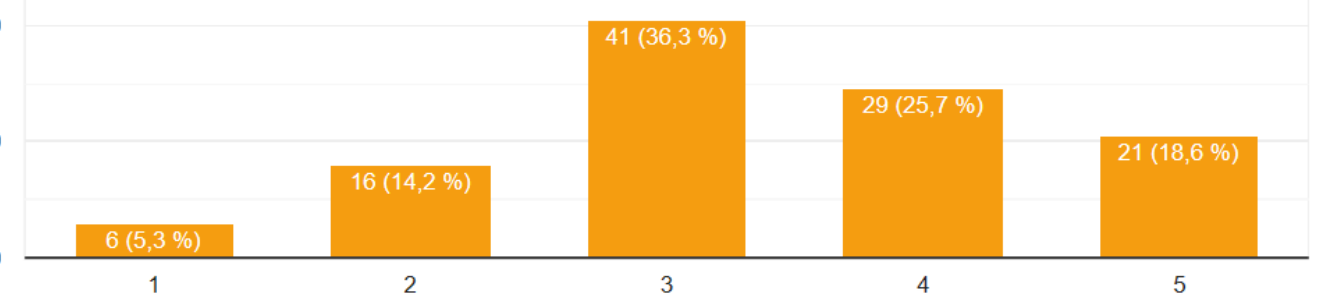

Fig. 13 Respuestas a la pregunta: ¿Te han parecido útiles y adecuadas las actividades realizadas en clase, para corregir o afianzar conocimientos?

En la Figura 14 se observan los resultados a la pregunta: ¿Las actividades realizadas en el aula te han sido de utilidad para alcanzar conocimientos sobre la materia que consideras que no habrías logrado mediante la docencia tradicional? En estas respuestas no se encuentra una clara relación puesto que tan solo un $41 \%$ ha opinado que con la metodología tradicional no habría alcanzado los mismos conocimientos que con la nueva metodología.

¿Las actividades realizadas en el aula te han sido de utilidad para alcanzar conocimientos sobre la materia que consideras que no habrías logrado mediante la docencia tradicional?

113 respuestas

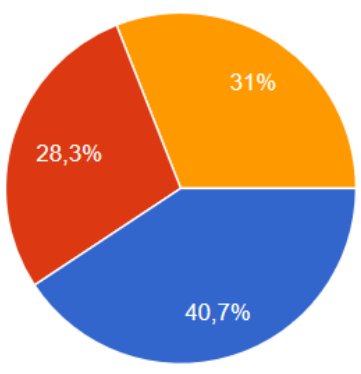

Fig. 14 Respuestas a la pregunta: ¿Las actividades realizadas en el aula te han sido de utilidad para alcanzar conocimientos sobre la materia que consideras que no habrías logrado mediante la docencia tradicional? 
En la Figura 15 se observan los resultados a la pregunta: ¿Consideras que has aprendido más con este nuevo tipo de metodología que en las clases en las que se emplea un método tradicional? De nuevo la dispersión de opiniones del grupo encuestado no decanta que ninguna de las metodologías les guste más o menos.

¿Consideras que has aprendido más con este nuevo tipo de metodología que en las clases en las que se emplea un método tradicional?

113 respuestas
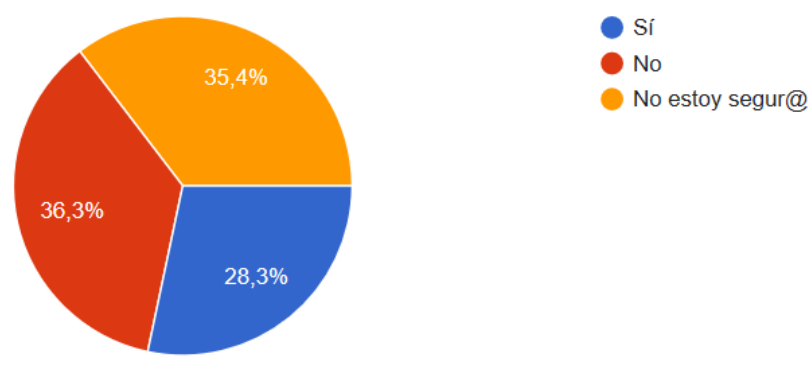

Fig. 15 Respuestas a la pregunta: ¿Consideras que has aprendido más con este nuevo tipo de metodología que en las clases en las que se emplea un método tradicional?

En la Figura 16 se observan los resultados a la pregunta: ¿Te gustaría que el resto de las sesiones fuesen también con la metodología de la docencia inversa? Las respuestas fueron muy claras y se pudo apreciar que a la mitad del grupo no les gusto la iniciativa de nuevo bien por la resistencia al cambio o quizás por la baja puntuación por la realización de las actividades en la nota final.

¿Te gustaría que el resto de sesiones fuesen también con la metodología de la docencia inversa?

113 respuestas
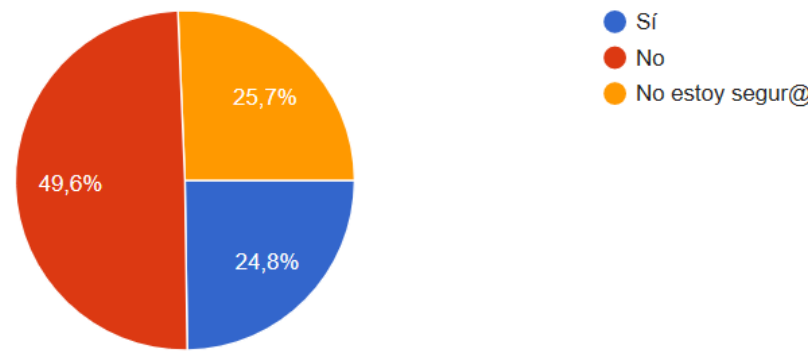

Fig. 16 Respuestas a la pregunta: ¿Te gustaría que el resto de las sesiones fuesen también con la metodología de la docencia inversa?

La última de las preguntas fue del tipo "respuesta corta" para poder permitir a los participantes dar su opinión al respecto y recoger sus feedback tanto positivos como negativos al respecto. Aunque no se muestran los resultados en este trabajo han sido los de mayor interés para poder extraer las conclusiones de esta experiencia. 


\section{Conclusiones}

Tras finalizar y analizar en más detalle los resultados tanto los académicos como los de la encuesta de satisfacción podemos concluir la implantación de la docencia inversa en las prácticas de la asignatura “Teoría y Diseño de Máquinas" ha sido una experiencia positiva pero no con los resultados esperados a pesar de la dedicación extra que se ha tenido que realizar tanto por parte del profesorado como del alumnado. A pesar de la resistencia al cambio de la metodología se puede apreciar como la participación en el proyecto podría aumentar si la puntuación por la realización de las actividades fuese mayor, por lo que nos pone en preaviso de que puede que no esté bien calibrado el esfuerzo del alumno con el valor de su puntuación en la nota final y se debería revisar para próximos cursos. Quizás puede que se deban poner de manifiesto cual es la intención de este tipo de actividades a pesar de su bajo porcentaje en la nota final su objetivo era la de adquirir unas competencias para poder realizar el trabajo final de la asignatura.

Respecto a los resultados académicos se puede apreciar cómo han mejorado considerablemente pero tampoco hay una clara relación que sea debido a la implantación de la docencia inversa o de otras iniciativas ya implantadas como la realización de tareas semanales para implicar y controlar más el aprendizaje continuo de los alumnos.

Respecto a los resultados de la encuesta de satisfacción es cierto que se realizó solamente terminar las sesiones iniciales de docencia inversa y quizás hubiese sido mejor esperar a realizar sesiones con el método tradicional para poder contrastar más las ventajas de la docencia. De todos modos, se pudo ver como en una muestra de 113 alumnos la diversidad de opiniones es grande y no todo el mundo tiene la misma predisposición a los cambios.

Para concluir comentar que a pesar de ese sabor agridulce por el gran esfuerzo realizado, los profesores hemos visto una posibilidad de mejora con la cantidad de comentarios que no remitieron en las encuestas de satisfacción y esperamos poder implantarlos los que se puedan en próximos cursos.

\section{Referencias}

BAS, M. D. C., SEgURA MAROTO, M., Ginestar Peiro, C. D. M., \& MAROto ÁlVAREZ, M. (2017). Preferencias en el aprendizaje de Investigación Operativa: Docencia inversa y presencial. Paper presented at the In-Red 2017. III Congreso Nacional de innovación educativa y de docencia en red.

BENÍTEZ, A. A., \& GARCÍA, M. L. (2013). Un primer acercamiento al docente frente a una metodología basada en proyectos. Formación universitaria, 6(1), 21-28.

FLIPPED CLASSROOM. (2019). Visión - What is the Flipped Classroom. Retrieved from https://www.theflippedclassroom.es/what-is-innovacion-educativa/

GARCÍA-CARPINTERO BLAS, E., SILES GONZÁLEZ, J., MARTÍNEZ ROCHE, M. E., MARTÍNEZ MIGUEL, E., MANSO PEREA, C., \& ÁlVAREZ EMBARBA, B. (2017). Metodologías de enseñanza-aprendizaje en enfermería:¿ Es el portafolio una metodología acorde con el Espacio Europeo de Educación Superior?

MONTAVA JORDÀ, S., SÁNCHEZ CABALLERO, S., SELLES CANTÓ, M. A., \& MARTÍNEZ SANZ, A. V. (2019). Implementación de las tareas semanales mediante la plataforma PoliformaT para la mejora de resultados en el aprendizaje por proyectos. Paper presented at the IN-RED 2019. V Congreso de Innovación Educativa y Docencia en Red.

SAMS, A., \& BERGMANN, J. (2013). Flip your students' learning. Educational leadership, 70(6), 16-20.

SÁNCHEZ, M. R. (2011). Metodologías docentes en el EEES: de la clase magistral al portafolio. Tendencias pedagógicas(17), 83-103.

(cc) EY-NC-ND 2020, Universitat Politècnica de València

Congreso In-Red (2020) 
UPV ASIC - ICE. (2018). Guía de implantación de la DOCENCIA INVERSA UPV,. Retrieved from www.upv.es/entidades/VRED/info/U0784192.pdf

UPV Docencia Inversa. (2014). PROYECTO DOCENCIA INVERSA UPV. Retrieved from https://docenciainversa.blogs.upv.es/proyecto-clase-inversa-upv/

UPV Vicerrectorado de Recursos Digitales y Documentación. (2018). Convocatoria A+D 2018-2019. Retrieved from http://www.upv.es/entidades/VRED/info/ConvocatoriaA+D2018.pdf 